\title{
Topic Transition Strategies for an Information-Giving Agent
}

\author{
Nadine Glas \\ Institut Mines-Télécom, Télécom ParisTech, \\ CNRS, LTCI \\ 46 Rue Barrault, 75013 Paris, France \\ glas@telecom-paristech.fr
}

\author{
Catherine Pelachaud \\ CNRS, LTCI, Télécom ParisTech \\ 46 Rue Barrault, 75013 Paris, France \\ pelachaude \\ telecom-paristech.fr
}

\begin{abstract}
We have explored how a conversational agent can introduce a selected topic in an ongoing non-task oriented interaction with a user, where the selected topic has little to do with the current topic. Based on the reasoning process of the agent we have constructed a set of transition strategies to introduce the new topic. We tested the effects of each of these strategies on the perception of the dialogue and the agent.
\end{abstract}

\section{Introduction}

The choice of the dialogue topics that an agent initiates in non-task oriented human-agent interaction is important for several aspects of the interaction such as the coherence of the interaction (Macias-Galindo et al., 2012) and the user's engagement (Glas and Pelachaud, 2015). Multiple efforts are oriented towards the selection of the appropriate topic at a specific point in the interaction. However, how the selected topic can or should be introduced by the agent has not been given much consideration in non-task oriented dialogue.

In this work we will explore the latter aspect by looking at utterances that may be used to initiate a transition from one topic to another. We shall call these utterances transition strategies. By comparing a selection of transition strategies we try to answer two questions: 1) What strategies have the potential of keeping/making the dialogue coherent? And 2) what effect do the use of the different transition strategies have on the perception of the conversational agent? The answers to these questions will serve to automatically generate agent strategies to connect one topic to another in non-task oriented dialogue.

\section{Domain}

This research is performed for the French project 'Avatar 1:1' that aims at developing a human-sized virtual agent in a museum. The goal of the agent is to engage human visitors in interaction about the artworks of the museum. In this informationgiving chat (Glas and Pelachaud, 2015) each artwork that is discussed is defined as a topic of the interaction. The discussion of an artwork's characteristic corresponds to a subtopic (Glas et al., 2015).

Previously, we found out that the topic of the interaction has an important influence on the user's level of engagement (Glas and Pelachaud, 2015). We had human users talk with a virtual agent that addressed several topics, correponding to different artworks. The users indicated that they were more engaged when the agent talked about artworks for which the users have a stronger preference (defined here as degree of liking) than when the agent talked about less preferred topics (Glas and Pelachaud, 2015). We are therefore working on an engagement driven topic manager that dynamically selects topics for the ongoing interaction taking into account the user's preferences (Glas et al., 2015). In the present work we are interested in agent strategies that may be used to connect the newly selected topic to the current topic of interaction. This is necessary as the topics are primarily selected according to their potential of engaging the user instead of their coherence with respect to the previous topic. Where other dialogue systems look at what topic is coherent at a specific point in the interaction (e.g. MacialGalindo et al., 2012; Breuing and Wachsmuth, 2012; Wong et al., 2012), we are looking at possible strategies to introduce a topic coherently.

\section{Related Work}

\subsection{Transition Strategies in Theory}

Literature about transition strategies outside taskoriented applications can be found in the domains of conversational analysis and social sci- 
ences, where they are studied from an observational (detection) point of view. Downing (2000) distinguishes two forms of introducing a topic: by means of an informative statement, and by asking a question. By informing the speaker assigns $\mathrm{him} /$ herself the role of topic supplier, whereas by questioning or eliciting a topic this role is offered to an interlocutor in the discourse.

Similarly, Button and Casey (1985) define two global ways of introducing a topic that is not related to the prior topic in a conversation: by a topic initial elicitor that is used to elicit a candidate topic from the next speaker while being mute with respect to what that topic may be, and by topic nominations that are oriented to particular newsworthy items. Two sequence types that may be used for topic nomination are itemised news enquires and news announcements. An itemised news inquiry is oriented to a recipient's newsworthy item where a news announcement is oriented to a speaker's newsworthy item.

Maynard and Zimmerman (1984) identified four topic initiation strategies in dyadic humanhuman conversations. For acquainted parties: displaying prior experience and using setting talk, and for unacquainted parties: categorisation question-answer pairs (e.g. year in school, academic major, etc.) and question-answer pairs involving activities that are related to the categories.

Hobbs (1990) focuses on three coherence relations that he claims are responsible for most of the so-called topic drift in dialogue: parallelism, explanation and metatalk. Parallelism between two segments occur when the two segments assert propositions from which we can infer that identical properties hold of similar entities, or that similar properties hold for identical entities. An explanation occurs when one segment functions as the explanation of a previous segment and metatalk asserts a relation between some segment and the goals of the conversation.

\subsection{Transition Strategies in Dialogue Systems}

To our knowledge, existing dialogue systems that explicitly consider different strategies to introduce a particular topic have been developed exclusively for task oriented interaction, in particular in the form of task interruption strategies. In this context McFarlane (2002) defines four primary methods: immediate, negotiated, mediated, and scheduled interruption. Yang et al. (2008) found out that dialogue partners usually use discourse markers and prosody cues to signal task switching. Guided by these works Heinroth et al. (2011) looked at 4 different task switching strategies: unassisted immediate topic shift, discourse markers combined with prosody cues, and two full sentence initialising topic shifts to produce a more natural dialogue flow and to increase the timespan the user has for task switching: explanation and negotiation strategies. The explanation strategy explains what task is about to be started and the negotiation strategy asks for permission to switch a task. They evaluated the use of these four strategies on several dimensions and found that the explanation strategy showed high scores regarding efficiency and userfriendless and supports the user to memorise the tasks. Other strategies showed advantages such as being less irritating.

\subsection{Guidelines for Topic Transitions}

The above mentioned research demonstrates that there does not exist one overall taxonomy of transition strategies that can be used as a recipe for transition strategy generation in non-task oriented dialogue. This lack shows the need of our own research towards transition strategies and makes us fall back to the following generally accepted ideas about topic switching: According to Clark (1996) a topic can be described as a joint project as it is jointly established during ongoing conversations. Svennevig (2000) adds that every spoken contribution may raise new potential topics whose actual realisation depends on the co-participant's acceptance by picking up one of these topics within his or her reply. To conclude, Sacks (1971, April 5 in: Levinson, 1983:313) made an overall remark that what seems to be preferred for a topic shift is that if A has been talking about X, B should find a way to talk about $Z$ (if $Z$ is the subject he wants to introduce) such that $X$ and $Z$ can be found to be natural fellow members of some category $Y$. In the current work we try to collect more precise indications about how to generate transition strategies in non-task oriented dialogue.

\section{Methodology}

In order to find out what strategies a conversational agent can use to initiate topic transitions in nontask oriented dialogue we follow Heinroth et al. (2011) (Section 3.2) by testing a set of potential transition strategies with respect to their effects on 


\begin{tabular}{|ll|c|}
\hline Speaker & Dialogue about "Luncheon on the Grass" by Claude Monet & Subtopic \\
\hline \multirow{2}{*}{ Agent: } & $\begin{array}{l}\text { [...] } \\
\text { Claude Monet was a French painter. He lived his entire life at Giverny, a beautiful village north } \\
\text { of Paris. }\end{array}$ & Artist \\
User: & $\begin{array}{l}\text { Yes I know. I visited Giverny last year. } \\
\text { Agent: }\end{array}$ & This painting was made around 1865. \\
User: & Yes, I've read so too. & Period \\
\hline
\end{tabular}

Table 1: An example of a dialogue fragment preceding a topic switch initiated by a transition strategy. In the experiment this dialogue fragment (translated) serves as the context of scenario 1 (Section 4.3).

the perception of the dialogue and the agent. In the subsections below we respectively discuss the steps to achieve this: the specification of the context of the transition strategies (Section 4.1), the design of the transition strategies themselves (Section 4.2), the setup of the experiment to test the set of strategies (Section 4.3), and the questionnaire that will be used for this (Section 4.4).

\subsection{Context of the Transition Strategies}

The strategies that have been mentioned in previous work vary with respect to the context. Some strategies work for topics that are interesting for the listener and others for those that are interesting for the speaker (Button and Casey, 1985). Some strategies are used by acquainted parties and others by unacquainted parties (Maynard and Zimmerman, 1984). Explanation strategies in the sense of Hobbs (1990), as well as metatalk only work for a specific set of topics.

These constraints imply that the strategies that can be used to introduce a topic in a conversation depend on the relation between the current topic of the dialogue and the new topic that is to be introduced. The first step in generating transition strategies is thus to define this relation. In the context of project Avatar 1:1 (Section 2) we are looking at strategies that an agent can employ in interaction with an unacquainted user to make the transition between two discussion phases about two different artworks. In the current work we will focus on what seems the most extreme case, namely the transition between discussion phases of two very different artworks: Artworks that have nothing in common except from the fact that they are both artworks in the same museum. In this way we test if the agent's topic manager can indeed be allowed the flexibility to select any given artwork of the museum as next topic of the discussion. Such flexibility helps finding (initiating) the topic that engages the user most (Glas et al., 2015).
To be more precise, in Table 1 we give an example of a dialogue fragment that proceeds the moment at which the new topic, corresponding to a very different artwork than the one discussed, is to be introduced. As the timing of introducing a new topic may have an influence on the perception of the topic switch (Clark, 1996) we limit this research to a topic switch that occurs after the conversation has addressed respectively the artist and the period of the former discussed artwork.

\subsection{Design of Potential Transition Strategies}

Due to the nature of the context we are dealing with, the potential transition strategies to introduce a discussion phase of another artwork are limited to the following categories from the literature: explanations in the sense of Heinroth et al. (2011), informative statements (Downing, 2000), itemised news enquires and news announcements (Button and Casey, 1985), categorisation question-answer pairs and question-answer pairs involving activities (Maynard and Zimmerman, 1984), and parallelism (Hobbs, 1990). It is however not prescribed how we could generate formulations for each of these detection-based categories for the context we are looking at. We thus base the manual creation of a set of potential transition strategies that belong to one or multiple of these categories, on the general guideline by Sacks (1971, Section 3.3).

According to Sacks (1971) we need to find a way to let the former (current) and the next (selected) topic be members of some category Y. We try to do this by (indirectly) referring to an element that is used in the agent's reasoning process to talk about the next topic. The agent disposes of a knowledge base that holds information about certain artworks from the museum. From this set of artworks it selects dynamically a new topic of discussion with the goal of maximising the user's engagement level, taking into account the characteristics of the artworks (e.g. period, artist), the 


\begin{tabular}{|c|c|c|c|}
\hline Nr. & Strategy & $\begin{array}{l}\text { Element in Topic Man- } \\
\text { ager }\end{array}$ & $\begin{array}{l}\text { Orien- } \\
\text { tation }\end{array}$ \\
\hline 1. & $\begin{array}{l}\text { Pol }(\text { Pref } A(i))==\operatorname{Pol}(\text { Pref } A(j)) \\
\text { E.g. I also like the Balloon Dog by Jeff Koons }\end{array}$ & Preferences Agent $(i, j)$ & Agent \\
\hline 2. & $\begin{array}{l}\text { (Pref } A(j)>\text { Pref } A(i)) \\
\text { E.g. Personally, I prefer the Balloon Dog by Jeff Koons }\end{array}$ & Preferences Agent $(i, j)$ & Agent \\
\hline 3. & $\begin{array}{l}\text { Association } A(i, j) \\
\text { E.g. This work reminds me of the Balloon Dog by Jeff Koons }\end{array}$ & Associations Agent $(i, j)$ & Agent \\
\hline 4. & $\begin{array}{l}(\operatorname{Pol}(\operatorname{PrefU}(i))==+) \rightarrow(\operatorname{Pol}(\operatorname{Pref} U(j))==+) \\
\text { E.g. If you like this work, maybe you also like the Balloon Dog by Jeff } \\
\text { Koons }\end{array}$ & Preferences User $(i, j)$ & User \\
\hline 5. & $\begin{array}{l}(\text { Pref } U(j)>\text { Pref } A(i)) \text { ? } \\
\text { E.g. Maybe you prefer the Balloon Dog by Jeff Koons. }\end{array}$ & Preferences User $(i, j)$ & User \\
\hline 6. & $\begin{array}{l}\text { Experience } A(i)+\text { Experience } A(j) \\
\text { E.g. I've also seen the Balloon Dog by Jeff Koons }\end{array}$ & $i, j$ in Knowledge Base & Agent \\
\hline 7. & $\begin{array}{l}\text { ExperienceU }(i)+\text { ExperienceU }(j) \text { ? } \\
\text { E.g. Have you also seen the Balloon Dog by Jeff Koons? }\end{array}$ & $i, j$ in Knowledge Base & User \\
\hline 8. & $\begin{array}{l}\exists(j) \wedge(j \neq i) \\
\text { E.g. Another artwork is the Balloon Dog by Jeff Koons }\end{array}$ & $i, j$ in Knowledge Base & Object \\
\hline 9. & $\begin{array}{l}\exists(j) \wedge(\text { Artist }(j) \neq \text { Artist }(i)) \\
\text { E.g. An artwork from another artist is the Balloon Dog by Jeff Koons }\end{array}$ & $\begin{array}{l}\text { Characteristics }(i, j) \text { in } \\
\text { Knowledge Base }\end{array}$ & Object \\
\hline 10. & $\begin{array}{l}\exists(j) \wedge(\operatorname{Period}(j) \neq \text { Period }(i)) \\
\text { E.g. An artwork from another period is the Balloon Dog by Jeff Koons }\end{array}$ & $\begin{array}{l}\text { Characteristics }(i, j) \text { in } \\
\text { Knowledge Base }\end{array}$ & Object \\
\hline
\end{tabular}

Table 2: Potential transition strategies to connect the discussion phases of two very different artworks (translated). $i$ is the current topic of the interaction and $j$ is the one to be introduced. $A=$ Agent, $U=$ User, $P o l=$ Polarity, Pre $f=$ Preference.

preferences of the user and the agent for an artwork (degree of liking), and the agent's associations (Glas et al., 2015). The set of potential transition strategies that we created by referring to these elements is listed in Table 2. For each of the strategies we formulated an agent utterance to realise the strategy.

Strategies 9 and 10 that insist on the (in this case contrasting) characteristics of the artworks are added as a reference to the strategies that we would use for the transition between artworks that have characteristics in common (the category $\mathrm{Y}$ ).

\subsection{Experimental Setup}

Inspired by the existing literature we have created a set of potential transition strategies for the context we are looking at. In order to verify if each of these strategies is suitable to be generated by the agent to switch the topic in the information-giving chat with the user we perform an empirical study. By means of an online questionnaire we test the effect that the different transition strategies have on the perception of the dialogue and the agent.

To this end we present each participant with 2 different dialogue fragments (i.e. contexts) consisting of agent utterances and simulated user in- puts (as e.g. Macias-Galindo et al., 2012). Each scenario is followed by 3 randomly assigned transition strategies, displayed next to each other. We do not show the utterances that may follow the transition strategies. In this way we do not show an acceptance or rejection of the topic by the user (Clark, 1996; Svennevig, 2000). Directly after each of the 3 transition strategies we ask the participants to answer several questions (Section 4.4). Appendix A shows a fragment of the website for this experiment. We use a written setup to allow the participants to consider multiple strategies at the same time in the same context, enabling crosscomparison and rereading as much as desired. Besides, in this way the judgements are not disturbed by unnatural text-to-speech realisations.

As mentioned before, the dialogue fragment that represent the former topic in the context and the topic that is addressed in the transition strategies (next topic) are about very different artworks. We test 2 topic pairs for each participant (i.e. 2 different scenarios) to anticipate possible effects that are due to individual characteristics of a particular context. Scenario 1 consists of the discussion of a painting by Monet (shown in table 1) followed by transition strategies introducing a 
statue by Jeff Koons (listed in Table 2). Scenario 2 consists of the discussion of a painting by Mondrian followed by transition strategies introducing David, the statue by Michelangelo. The alternation of agent-user utterances, the number of utterances and the order of the subtopics are the same in both context fragments. The order in which the scenarios are presented to the participants is random. Pictures of the artworks next to the questionnaire make sure that all the participants know what the artworks look like (Appendix A).

\subsection{Questionnaire}

For each of the 3 selected transition strategies we ask questions on a scale from 1-9 (shown in Appendix A) (following Bickmore and Cassell, 2005). The first 3 questions relate to the perception of the dialogue and serve to answer the first question we try to answer (Section 1): What strategies have the potential of keeping/making the dialogue coherent? We ask respectively if the participant finds the dialogue natural (Nakano and Ishii, 2010; Bickmore and Cassell, 2005), coherent (Macias-Galindo et al., 2012), and smooth (Higashinaka et al., 2008; Nakano and Ishii, 2010).

The following 5 questions serve to answer our second question (Section 1): What effect do the use of the different transition strategies have on the perception of the conversational agent? We ask respectively to what extent the participants find the agent friendly, warm, fun (in French "stimulant"), competent and informed (in French "cultivé) (Bickmore and Cassell, 2005). These measures are related to 2 important social aspects, warmth and competence (Fiske et al., 2007).

\section{Results}

83 subjects filled out the questionnaire: 56 female, all native speakers of French, aged 19-69. In the subsections below we show the results of the experiment specified for the two issues we are looking at: the perception of the dialogue and the perception of the agent.

\subsection{Dialogue Perception}

For each strategy, the perception of the dialogue has been questioned for the two scenarios and on three dimensions: naturalness, coherence, and smoothness. For each of these dimensions the results show no significant difference between the two scenarios (Kruskal-Wallis). This means that we can take the data for both scenarios together, as shown in Figures 1, 2, and 3.

For all three dimensions the scores differ significantly among the strategies (Kruskal-Wallis $p<0.01)$. Regarding the level of naturalness and smoothness, Kruskal-Wallis multiple comparisons show that the significant differences are due to the strategies 9 and 10 that score significantly lower than some others, indicated by the horizontal brackets in the graphs. Regarding the coherence of the dialogue, strategy number 10 leads to a significantly lower level than other strategies.

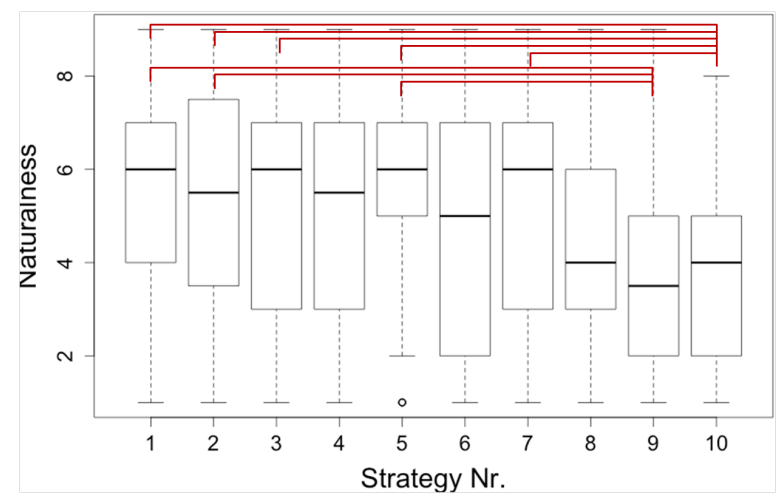

Figure 1: Naturalness for each strategy, $p<0.01$.

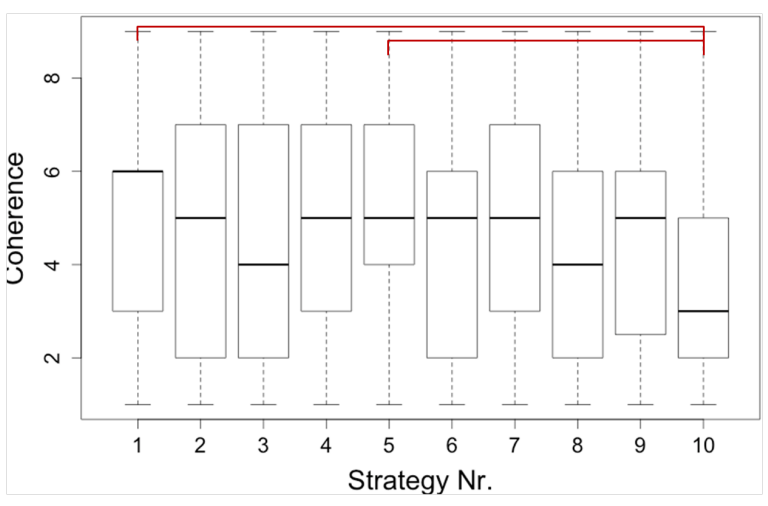

Figure 2: Coherence for each strategy, $p<0.01$.

Only strategies 1 to 7 show a higher mean than the average level (4.5 on a scale of 9) on the dimensions of naturalness and smoothness. With respect to the level of coherence, except from strategies the 8,9 and 10, strategy 3 also scores lower than average (mean).

As mentioned in Section 4.2 the strategies are either oriented towards the agent, the user, or the (characteristics) of the object (artwork). The strategies from the latter group lead to significantly lower levels of naturalness, coherence 


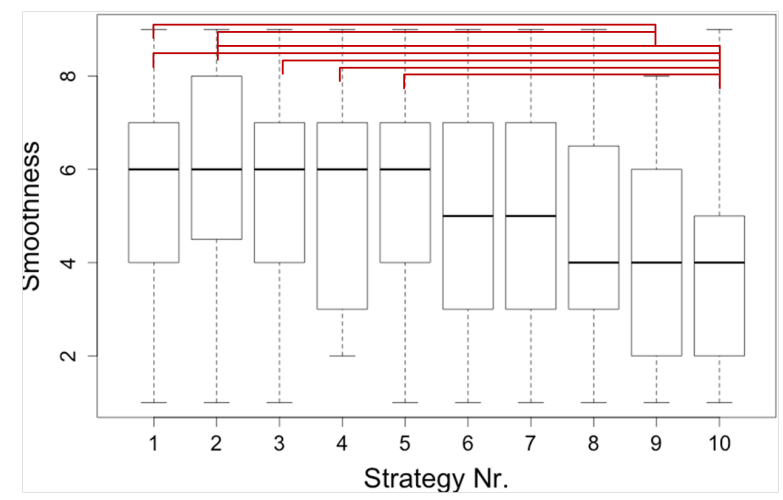

Figure 3: Smoothness for each strategy, $p<0.01$.

and smoothness in comparison to the strategies with another orientation (both scenarios, KruskalWallis $p<0.01)$. There is no significant difference in the scoring of the strategies that are agent oriented versus the ones that are user oriented with respect to the perception of the dialogue.

\subsection{Agent Perception}

Questions 4 to 8 are about the way the participants of the experiment perceive the social competence (Fiske et al., 2007) of an agent that would use the transition strategies in the context in which they are presented. The results show that between the two scenarios, the participants find the agent not significantly different with respect to its level of friendliness and knowledge ("informed") (Kruskal-Wallis). For these dimensions we can thus analyse the data for both scenarios together. Figure 4 and 5 specify the distributions of these dimensions for every strategy. The level of friendliness differs significantly among the strategies (Kruskal-Wallis $p<0.01$ ), which is due to strategies 8, 9, and 10 (Kruskal-Wallis multiple comparisons). However, only strategy 10 scores below average for the level of friendliness (mean $<4.5$ ). For all strategies the agent is not perceived significantly different with respect to its knowledge ("informed") and all of the strategies score above average on this dimension (mean $<4.5$ ).

In contrast to the agent's level of friendliness and knowledge ("informed"), for the dimensions of warmth, fun and competence, some strategies are significantly differently judged among both scenarios (Kruskal-Wallis $p<0.05$ ). Figure 6, 7 and 8 show the distribution of the results specified for both scenarios. The circled numbers indicate the strategies that are judged differently between

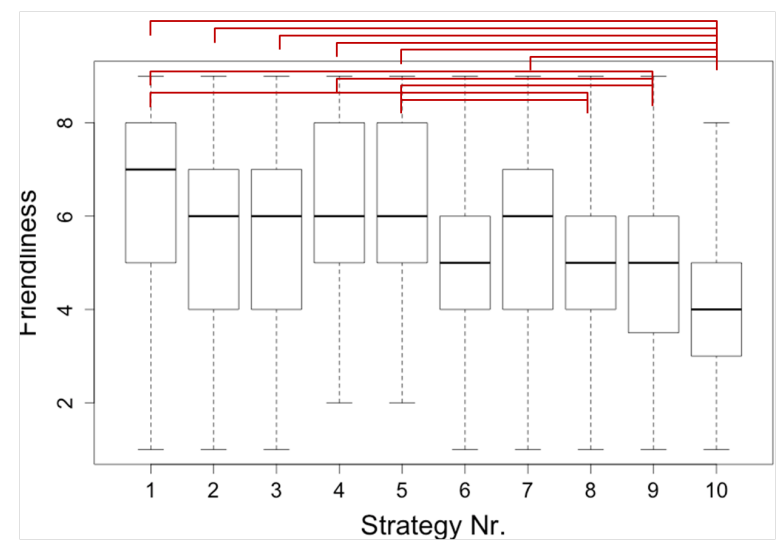

Figure 4: Friendliness for each strategy, $p<0.01$.

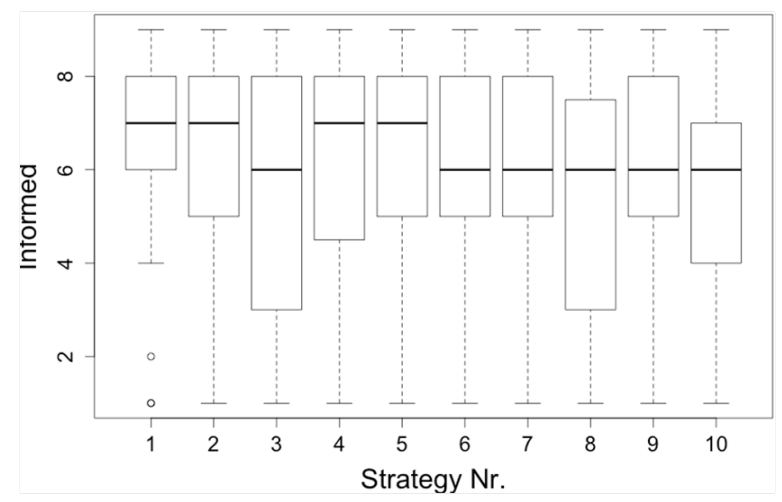

Figure 5: Agent being informed for each strategy.

both scenarios. On the dimension of warmth, strategy number 8 scores significantly higher in the second scenario (Monet-Koons) than in the first (Mondian-Michelangeloo). Together with strategy 6, strategy 8 also scores higher in the second scenario with respect to the level of fun that the participants perceived in the agent. Further, in the second scenario strategies 4 and 6 score higher on the dimension of competence than in the first scenario.

With respect to the agent's perceived level of warmth as well as fun, in scenario 1, strategies 8,9 and 10 score significantly lower than other strategies (Kruskal-Wallis $p<0.01$ ). In this scenario strategies 6,8 and 10 also score below average (mean $<4.5$ ). For scenario 2 strategy 10 scores significantly lower than other strategies (KruskalWallis $p<0.01)$ and falls below average.

Scenario 1 shows significant differences between the scorings of the agent's perceived level of competence (Kruskal-Wallis $p<0.01$ ). Multiple comparisons (Kruskal-Wallis) do not indicate a specific pair of strategies that is responsible for this difference. Strategy 3 is the only strategy that 


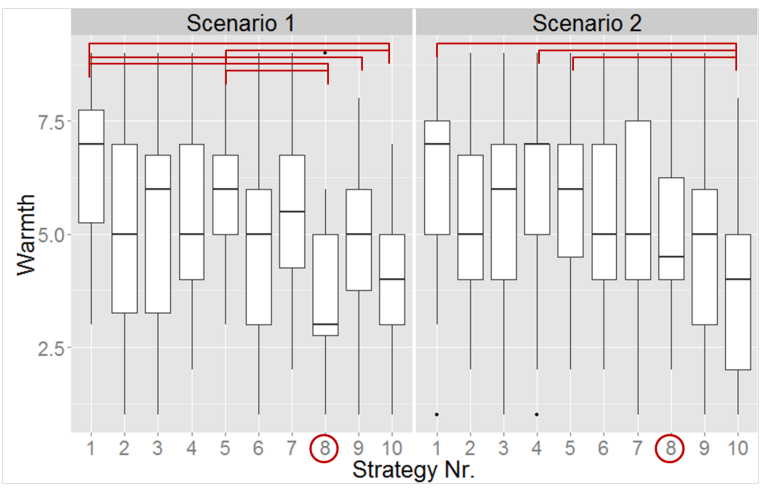

Figure 6: Warmth for each strategy, $p<0.01$. Between scenarios strategy 8 differs $p<0.05$.

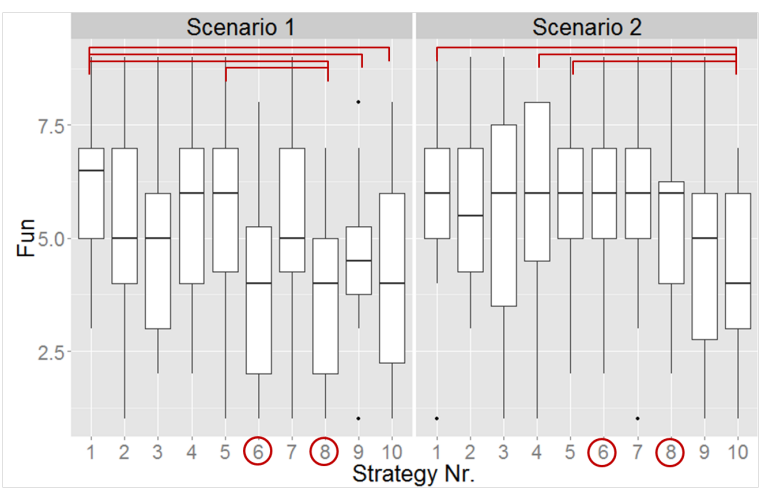

Figure 7: Fun for each strategy, $p<0.01$. Between scenarios strategies $6, p<0.05$, and 8 , $p<0.01$, differ.

scores below average (mean $<4.5$ ). In scenario 2 the strategies show no significant differences or scorings below average.

Comparing the strategies that are oriented towards the agent with those that oriented towards the user (Table 2) does not lead to a significant difference with respect to the perception of the agent (both scenarios, Kruskal-Wallis). The strategies that are not oriented towards the agent or user, but refer to the (characteristics) of the object (artwork) lead to significantly lower levels of friendliness, warmth and fun in comparison with the strategies that are oriented towards the interaction participants (both scenarios, Kruskal-Wallis $p<0.01$ ).

Within the group of strategies that are agent or user oriented, we can make another grouping according to the element of the agent's reasoning process that is referred to: preferences $(1,2,4,5)$, associations (3) and the presence of an artwork in the agent's knowledge base $(6,7)$. A comparison between these groups leads to one significant re-

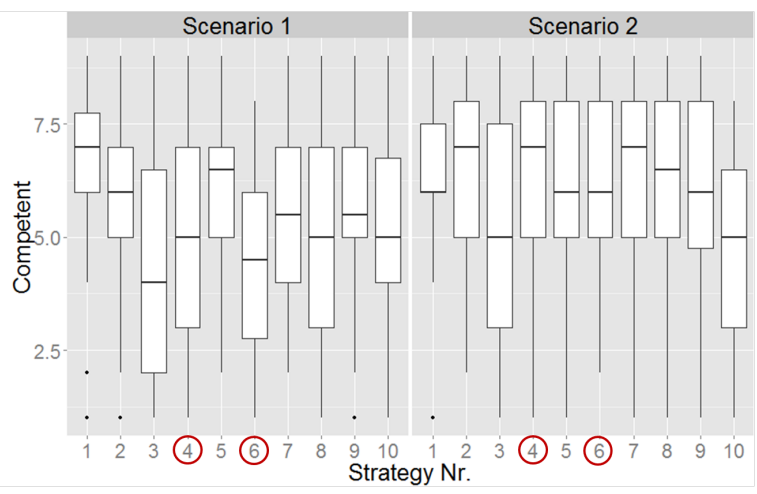

Figure 8: Competence for each strategy, $p<0.01$ for scenario 1 . Between scenarios strategies $4, p<$ 0.05 , and $6, p<0.01$, differ.

sult: in scenario 1, strategies that use preferences score significantly higher on competence than the strategies from the other groups (Kruskal-Wallis $p<0.01$, Kruskal-Wallis multiple comparisons).

\section{Discussion}

The results show that the transition strategies that insist on contrasting characteristics of the artworks such as a different artist (strategy 9) or period (strategy 10) lead to scores below average (4.5 on a scale of 9) with respect to the level of naturalness, coherence and smoothness. On the level of naturalness and smoothness the difference with the other strategies is significant. This demonstrates that even when the artist and period of the former artwork have been discussed just before the transition strategies, making transition strategies that are based on (referring back to) the earlier discussed characteristics (subtopics), does not guarantee a natural, coherent and smooth dialogue.

The fact that strategy 8 , that presents another artwork as just being another artwork, scores below average on naturalness and coherence, shows that "being an artwork" is not a category that can sufficiently bind both topics ("category Y" by Sacks, 1971 in Levinson, 1983). This can have several reasons: The transition strategy may not succeed in presenting the former and latter artwork in being natural fellow members of some category $\mathrm{Y}$, in this case being an artwork. Category $\mathrm{Y}$ may need to be a more restrictive (distinctive) category than one to which all topics belong (all topics are artworks) in order to bind two specific topics. Or, making both topics natural fellow members of some category Y may not be sufficient 
in general to establish a natural and coherent dialogue.

A reason why strategy 3 that is based on the associations of the agent, scores bad on coherence but well on naturalness and smoothness may be due to the fact that the participants can find the association itself incoherent. Due to the contrasting characteristics of the artworks the participants may find it incoherent that the first artwork reminds the agent of the second. However, given that the strategy is considered natural and smooth implies that it might be a suitable strategy to connect the discussions of two similar artworks.

The same explanation can be given for the low scoring of this strategy (3) with respect to the perception of the agent's level of competence (scenario 1). When the agent associates two artworks that do not seem alike the agent is perceived less competent than average.

The strategies that lead to low scores on naturalness, coherence and smoothness of the dialogue $(8,9$ and 10$)$ also score relatively low with respect to the perception of friendliness, fun and warmth of the agent. This gives us reasons to suspect that both aspects are related: when a dialogue is not considered natural, coherent or smooth, the agent is not considered as very friendly, fun and warm.

The participants do not perceive the agent significantly more or less informed when it uses certain transition strategies instead of others. This shows that referring explicitly to the characteristics of the artworks such as its artist (9) or period (10) does not make the agent look more informed than when the strategies refer to more subjective aspects of the agent's reasoning process, such as its preferences or associations.

On the contrary, strategies that refer to the preferences of the interaction participants score significantly higher with respect to the agent's level of competence than the strategies that use other variables from the agent's reasoning process.

With respect to the consequences of the transition strategies on the perception of the dialogue the results have shown no significant difference among both scenarios. The effects on the perception of the dialogue that are discussed in this Section seem thus generalisable for the domain we are looking at (Section 4.1). However, for some transition strategies the perception of the agent is judged significantly differently among the two scenarios. For example, strategy 6, a statement of the fact that the agent has seen some other artwork, has in some contexts a negative influence on the agent's level of fun and competence, where this is not the case in other contexts. In the two scenarios that were used for this experiment the type of information, the utterance types, and the number of utterances are equal. Therefore, further research will be needed to show what exactly the underlying reason is that the same strategies lead, in a different scenario, to a difference in the perception of the agent.

\section{Conclusion}

In this work we have looked at how a selected topic of discussion can be introduced by an agent in an ongoing non-task oriented dialogue. In the context we are looking at, each topic consists of the discussion of an artwork from a museum. Inspired by social and conversational analytic literature we first constructed a set of candidate transition strategies. We then checked the consequences of each of these transition strategies on the perception of the dialogue and the agent.

We have found that the strategies that score well on all dimensions and all tested circumstances are those that ask for the experience of the user, and those that refer to the preferences of the interaction participants. Whether the preference is the agent's or the user's, and whether or not the new topic is preferred over the current one, transition strategies that integrate any type of preference maintain the coherence of the dialogue while maintaining/establishing a positive perception of the agent. The fact that certain transition strategies can connect topics about very different artworks while maintaining positive perceptions of the dialogue and the agent, shows that the agent's topic manager can indeed be allowed to select any topic required to engage the user at any moment in the conversation (Glas et al., 2015).

We plan to use the observations we obtained in this study by automatically generating appropriate transition strategies for the conversational agent whenever the topic manager initiates a topic switch. The automatic generation of the transition strategies could be performed by means of templates where the object names and characteristics can be generated from the agent's knowledge base. In the future we would like to explore the effects of the timing of the topic switch on the perception of the topic transition (Clark, 1996). Lastly, 
we would like to consider the agent's non-verbal behaviour with respect to topic switching. Nonverbal behaviour plays an important role in topic switching (Kendon, 1972) and in the perception of verbal behaviour in general (Sidner et al., 2005).

\section{Acknowledgements}

We would like to thank Sophie Rosset and Andrew Kehler for valuable discussion, and Brice Donval and Caroline Langlet for technical support. We would also like to thank all the participants of the experiment. This research is partially funded by the French project Avatar 1:1, ANR MOCA, and the Labex SMART (ANR-11-LABX65) supported by French state funds managed by the ANR, within the Investissements d'Avenir program under reference ANR-11-IDEX-0004-02.

\section{References}

Timothy Bickmore and Justine Cassell. 2005. Social dialongue with embodied conversational agents. In Advances in natural multimodal dialogue systems, pages 23-54. Springer.

Alexa Breuing and Ipke Wachsmuth. 2012. Let's talk topically with artificial agents! providing agents with humanlike topic awareness in everyday dia$\log$ situations. In Proceedings of the 4th international conference on agents and artificial intelligence (ICAART), volume 2.

Graham Button and Neil Casey. 1985. Topic nomination and topic pursuit. Human studies, 8(1):3-55.

Herbert H Clark. 1996. Using language, volume 1996. Cambridge university press Cambridge.

Angela Downing. 2000. Talking topically. CIRCLE of Linguistics Applied to Communication (CLAC), 3:31-50.

Susan T Fiske, Amy JC Cuddy, and Peter Glick. 2007. Universal dimensions of social cognition: Warmth and competence. Trends in cognitive sciences, 11(2):77-83.

Nadine Glas and Catherine Pelachaud. 2015. User engagement and preferences in information-given chat with virtual agents. In Workshop on Engagement in Social Intelligent Virtual Agents. Forthcoming.

Nadine Glas, Ken Prepin, and Catherine Pelachaud. 2015. Engagement driven topic selection for an information-giving agent. In Workshop on the Semantics and Pragmatics of Dialogue (SemDial), pages $48-57$.
Tobias Heinroth, Savina Koleva, and Wolfgang Minker. 2011. Topic switching strategies for spoken dialogue systems. In INTERSPEECH, pages 20772080.

Ryuichiro Higashinaka, Kohji Dohsaka, and Hideki Isozaki. 2008. Effects of self-disclosure and empathy in human-computer dialogue. In Spoken Language Technology Workshop, 2008. SLT 2008. IEEE, pages 109-112. IEEE.

Jerry R Hobbs. 1990. Topic drift. Conversational organization and its development, 38:3-22.

Adam Kendon. 1972. Some relationships between body motion and speech. Studies in dyadic communication, 7:177.

Stephen C Levinson. 1983. Pragmatics (Cambridge textbooks in linguistics). Cambridge University Press.

Daniel Macias-Galindo, Wilson Wong, John Thangarajah, and Lawrence Cavedon. 2012. Coherent topic transition in a conversational agent. In Proceedings of the 13th Annual Conference of the International Speech Communication Association (InterSpeech), Oregon, USA.

Douglas W Maynard and Don H Zimmerman. 1984. Topical talk, ritual and the social organization of relationships. Social psychology quarterly, pages 301-316.

Daniel McFarlane. 2002. Comparison of four primary methods for coordinating the interruption of people in human-computer interaction. Human-Computer Interaction, 17(1):63-139.

Yukiko I Nakano and Ryo Ishii. 2010. Estimating user's engagement from eye-gaze behaviors in human-agent conversations. In Proceedings of the 15 th international conference on Intelligent user interfaces, pages 139-148. ACM.

Candace L Sidner, Christopher Lee, Cory D Kidd, Neal Lesh, and Charles Rich. 2005. Explorations in engagement for humans and robots. Artificial Intelligence, 166(1):140-164.

Jan Svennevig. 2000. Getting acquainted in conversation: a study of initial interactions, volume 64. John Benjamins Publishing.

Wilson Wong, Lawrence Cavedon, John Thangarajah, and Lin Padgham. 2012. Flexible conversation management using a bdi agent approach. In Intelligent Virtual Agents, pages 464-470. Springer.

Fan Yang, Peter A Heeman, and Andrew Kun. 2008. Switching to real-time tasks in multi-tasking dialogue. In Proceedings of the 22nd International Conference on Computational Linguistics-Volume 1, pages 1025-1032. Association for Computational Linguistics. 


\section{Appendix A. Website for the experiment}
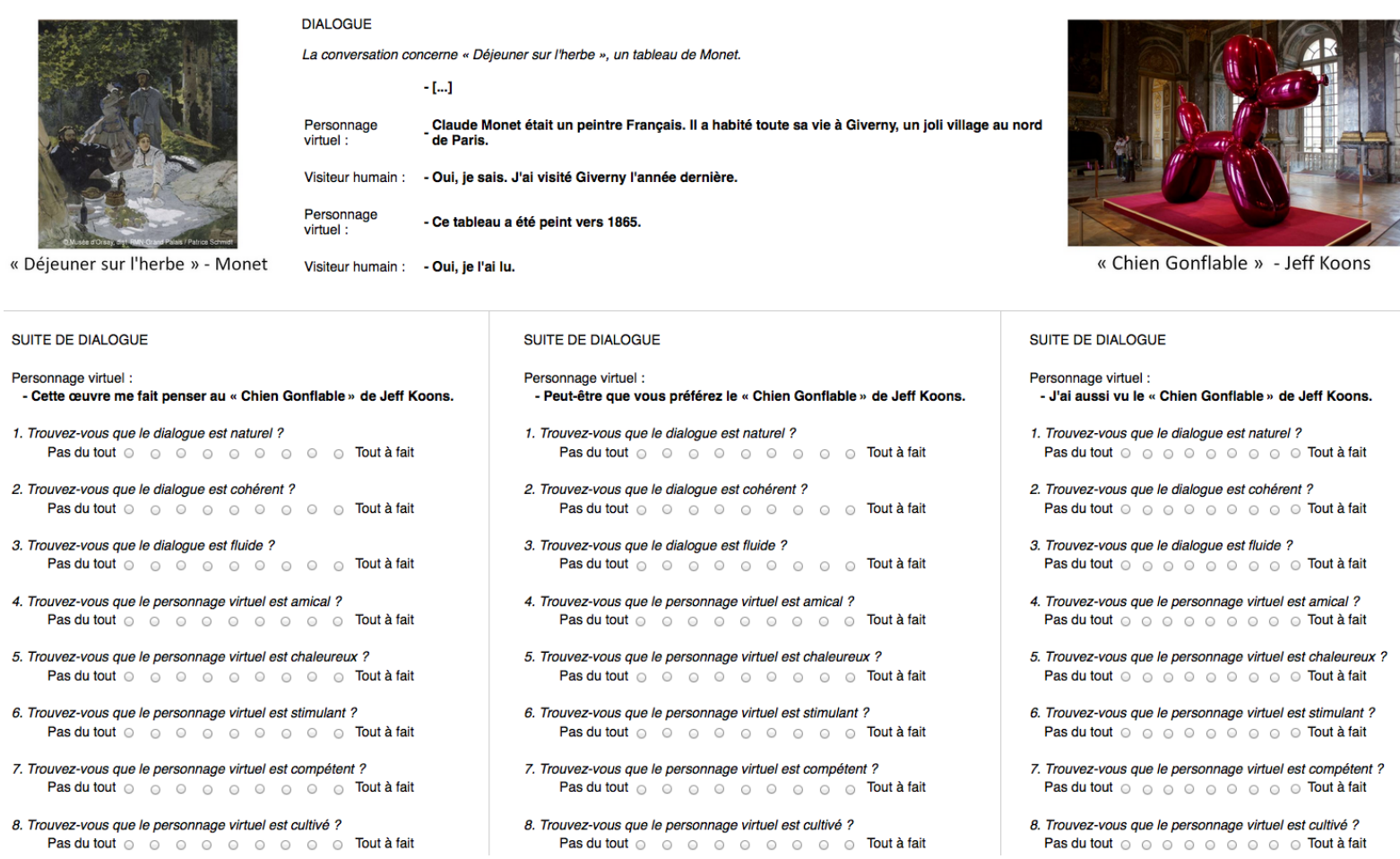

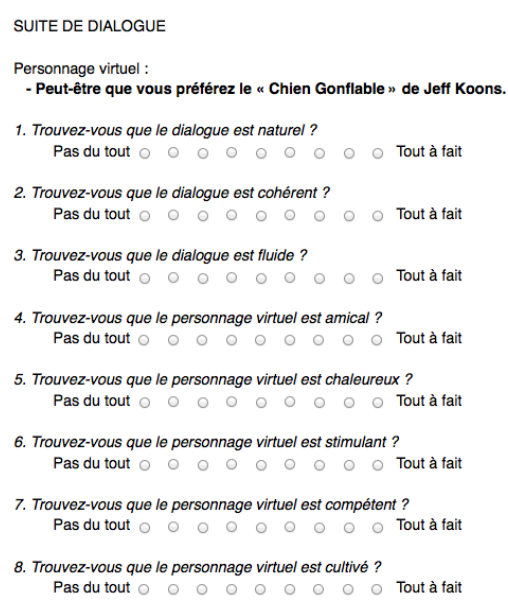

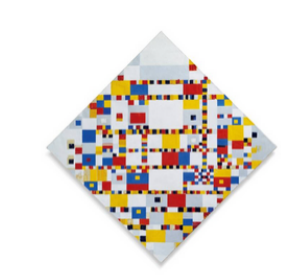

" Victory Boogie-Woogie » - Mondrian

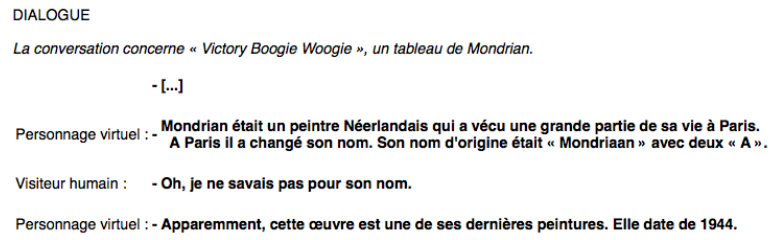

SUITE DE DIALOGUE Personnage virtuel - J'ai aussi vu le " David " de Michelangelo.

1. Trouvez-vous que le dialogue est naturel ? Pas du tout $\circ \circ \circ \circ \circ \circ \circ \circ \circ$ Tout à fai 2. Trouvez-vous que le dialogue est cohérent ? Pas du tout $\circ \circ \circ \circ \circ \circ \circ \circ \circ$ Tout à fait

3. Trouvez-vous que le dialogue est fluide ?

Pas du tout 000 qualogue est fluide?

4. Trouvez-vous que le personnage virtuel est amical ? Pas du tout $\circ \circ \circ \circ \circ \circ \circ \bigcirc \circ$ Tout à fait 5. Trouvez-vous que le personnage virtuel est chaleureux ?
Pas du tout $\bigcirc \circ \circ \bigcirc \circ \bigcirc \circ$ Tout à fait

6. Trouvez-vous que le personnage virtuel est stimulant ?
Pas du tout $\bigcirc \circ \circ$ Tout à fait

7. Trouvez-vous que le personnage virtuel est compéten ? Pas du tout $\triangle \circ \bigcirc \circ \circ \bigcirc \circ \bigcirc \circ$ Tout à fait

8. Trouvez-vous que le personnage virtuel est cultivé ?
"Chien Gonflable » - Jeff Koons

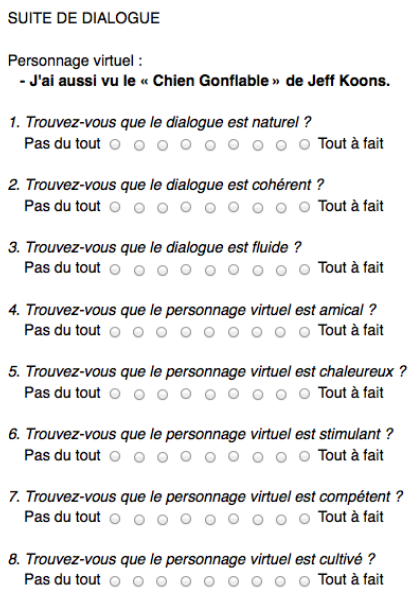

SUITE DE DIALOGUE

Personnage virtuel :

1. Trouvez-vous que le dialogue est naturel ?

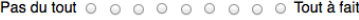

2. Trouvez-vous que le dialogue est cohérent ?

Pas du tout $\odot \circ \circ \bigcirc \circ \circ \circ \bigcirc \circ$ Tout à fait

3. Trouvez-vous que le dialogue est fluide ?

Pas du tout $\bigcirc \circ \circ \circ \circ \circ \circ \circ \circ$ Tout à fait

4. Trouvez-vous que le personnage virtuel est amical ?
Pas du tout

5. Trouvez-vous que le personnage virtuel est chaleureux ? Pas du tout $\bigcirc \circ \circ \bigcirc \circ \bigcirc \bigcirc \circ \bigcirc$ Tout à fait

6. Trouvez-vous que le personnage virtuel est stimulant ? Pas du tout $\bigcirc \circ \circ \circ \circ \bigcirc \circ \circ \bigcirc$ Tout à fait

7. Trouvez-vous que le personnage virtuel est compétent ?

Pas du tout $\bigcirc \circ \circ \quad 0$

8. Trouvez-vous que le personnage virtuel est cultivé ?
Pas du tout

\footnotetext{
Pas du tout $\triangle \circ$

8. Trouvez-vous que le personnage virtuel est cultivé ? Pas du tout $\circ \circ \quad \circ \quad \circ \quad \circ \quad \circ \quad \circ \quad \circ \quad \circ$ Tout à tait
}

Trouvez-vous que le personnage virtuel est compétent?

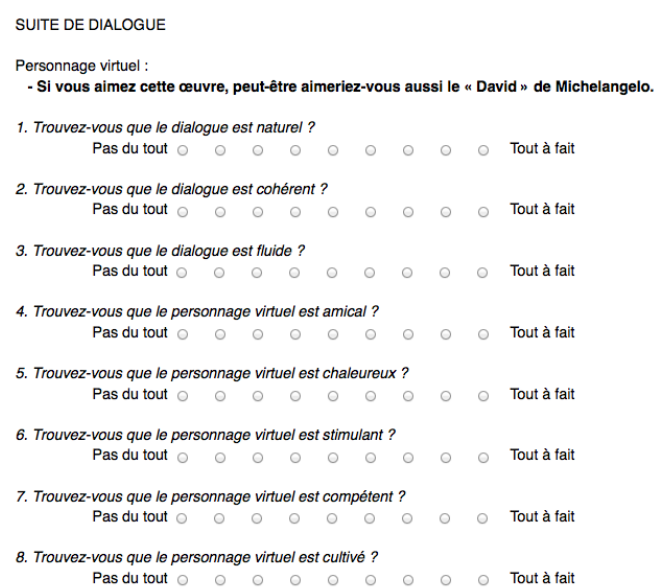

Figure 9: A screenshot of the website for the online experiment. The order of the scenarios and the selection of transition strategies differ among participants. 